Pacific Journal of Mathematics

APPROXIMATION BY HOLOMORPHIC FUNCTIONS ON 


\title{
APPROXIMATION BY HOLOMORPHIC FUNCTIONS ON CERTAIN PRODUCT SETS IN $C^{N}$
}

\author{
BARNET M. WEINSTOCK
}

In this paper we prove several theorems concerning approximation by holomorphic functions on product sets in $C^{n}$ where each factor is either a compact plane set or the closure of a strongly pseudoconvex domain. In particular we show that every continuous function which is locally approximable by holomorphic functions on such a set is globally approximable. Our results depend on a generalization of a theorem of Andreotti and Stoll on bounded solutions of the inhomogeneous Cauchy-Riemann equations on certain product domains.

1. Statement of results. If $K$ is a compact set in $C^{n}$ let $C(K)$ denote the Banach space of continuous complex-valued functions on $K$ with the uniform norm, and let $H(K)$ denote the closure in $C(K)$ of the space of functions which are holomorphic in some neighborhood of $K$. When $n=1$, each function in $H(K)$ is the uniform limit of a sequence of rational functions which are finite on $K$ and the spaces $H(K)$ (usually denoted $R(K)$ in this instance) have been extensively studied. In particular, the following properties of $H(K)$ are wellknown in the case $n=1$ (cf. Chapter 3 of [2]):

(1) If $U$ is a neighborhood of $K, f \in C^{1}(U)$, and $\partial f / \partial \bar{z} \equiv 0$ on $K$, then $f \mid K \in H(K)$.

(2) If $f \in C(K)$ and if for each $x \in K$ there is a neighborhood $U_{x}$ of $x$ in $C$ such that $f \in H\left(K \cap \bar{U}_{x}\right)$, then $f \in H(K)$.

(3) If $\mu$ is a complex Borel measure on $K$, then $\mu=\partial \hat{\mu} / \partial \bar{z}$ where

$$
\hat{\mu}(z)=-\frac{1}{\bar{\pi}} \int_{K}(\zeta-z)^{-1} d \mu(\zeta)
$$

is locally integrable on $C . A$ measure $\mu$ is an annihilating measure for $H(K)$ (i.e., $\int f d \mu=0$ for all $\left.f \in H(K)\right)$ if and only if $\hat{\mu}$ is supported on $K$.

Properties (1)-(3) are not valid for arbitrary compact sets of $C^{n}$, even if one restricts one's attention to holomorphically convex, or even polynomially convex sets. A celebrated example of Kallin [6] shows that (2) fails in general for polynomially convex compact sets. Also, Chirka [3], by modifying her example, has shown that for each positive integer $s$ there is a compact holomorphically convex set $K^{s}$ in $C^{3}$ and a function $f_{s} \in C^{\infty}\left(K^{s}\right)$ such that $f_{s} \notin H\left(K^{s}\right)$ although $\bar{\partial} f_{s}$ 
vanishes on $K^{s}$ to order $s .^{1}$

In this paper we consider compact sets $K \subset C^{n}$ of the form $K=$ $K_{1} \times \cdots \times K_{r}$ where each $K_{i}$ is either a compact set in $C$ or the closure of a strongly pseudo-convex domain in $C^{n_{i}}$. For such $K$ we prove the following theorems:

THEOREM 1.1. If $U$ is a neighborhood of $K, f \in C^{r+1}(U)$, and $\partial^{\alpha} f / \partial \bar{z}^{\alpha} \equiv 0$ on $K$ for all $\alpha=\left(\alpha_{1}, \cdots, \alpha_{n}\right)$ with $\sum \alpha_{j} \leqq r$, then $f \in H(K)$.

Theorem 1.2. If $f \in C(K)$ and if for each $x \in K$ there is a neighborhood $U_{x}$ such that $f \in H\left(K \cap \bar{U}_{x}\right)$, then $f \in H(K)$.

THEOREM 1.3. A measure $\mu$ on $K$ is an annihilating measure for $H(K)$ if and only if there exist distributions $\lambda_{1}, \cdots, \lambda_{n}$ of order $\leqq r-1$ with support in $K$ such that $\mu=\sum \partial \lambda_{j} / \partial \bar{z}_{j}$.

It is possible that Theorem 1.1 remains valid if $f$ is merely required to satisfy $\partial f / \partial \bar{z}_{j} \equiv 0$ on $K, 1 \leqq j \leqq n$. We know of no counterexample.

Theorem 1.2 implies an approximation theorem of the KeldyshMergelyan type if, in addition to the above hypotheses, $K$ has the "segment property", i.e., if there is an open cover $\left\{U_{i}\right\}$ of $\partial K$ and corresponding vectors $\left\{w_{i}\right\}$ such that for $0<t<1 z+t w_{i}$ lies in the interior of $K$ whenever $z \in K \cap U_{i}$. In this case every function which is continuous on $K$ and holomorphic in the interior of $K$ satisfies the hypotheses of Theorem 1.2 so lies in $H(K)$. In particular, if $K$ is a product of smoothly bounded domains, then $K$ has the segment property. The case $r=1$ when $K$ is the closure of a strongly pseudoconvex domain in $\boldsymbol{C}^{n}$ has been treated by Lieb [8] and Kerzman [7]. We use their method to prove Theorem 1.2.

If we consider Theorem 1.3 in the case $r=1$ we conclude that each annihilating measure for $H(K)$ where $K$ is the closure of a strongly pseudo-convex domain is the $\bar{z}$-divergence of an $n$-tuple of measures supported on $K$ (distributions of order 0 ). This implies the following localization theorem for annihilating measures which is wellknown in case $n=1$ [2, Lemma 3.2.11] and which is a sort of dual version of Theorem 1.2, which it clearly implies.

THEOREM 1.4. Let $K$ be the closure of a strongly pseudoconvex domain in $\boldsymbol{C}^{n}$. Let $\mu$ be an annihilating measure for $H(K)$. If $\left\{U_{i}\right\}$ is a finite open covering of $K$ there exist annihilating measures

1 The referee has pointed out that Kallin, in an unpublished remark and without knowledge of Chirka's paper, observed that her counterexample could be made to yield this additional property. 
$\mu_{i}$ for $H\left(K \cap \bar{U}_{i}\right)$ (in particular each $\mu_{i}$ is supported in $U_{i}$ ) such that $\mu=\sum \mu_{i}$.

All of our results are derived from an estimate (Theorem 2.2 below) for Cauchy-Riemann operator in certain product domains. This theorem is a generalization of a theorem of Andreotti and Stoll [1] for the case of a polycylinder. Our proof, like theirs, follows the induction procedure used in the proof of the familiar Dolbeaut-Grothendieck lemma, but we make essential use of the representation theorem of Grauert and Lieb [5] for bounded solutions of the Cauchy-Riemann equations in strongly pseudo-convex domains.

\section{The basic estimate.}

DEFINITION 2.1. An open set $G$ in $C^{n}$ is called admissible if (a) $n=1$ and $G$ is a bounded open set or (b) $n>1$ and $G$ is a strongly pseudo-convex domain with $C^{\infty}$ boundary.

The following theorem is due to Grauert and Lieb [5] in the case $n>1$, and is simply a restatement of known properties of the Cauchy kernel when $n=1$.

THEOREM 2.1. Let $G$ be an admissible open set in $C^{n}$. Then there exists a differential form $\Omega(\zeta, z)$ of type $(n, n-1)$ in $\zeta$, of type $(0,0)$ in $z$, defined in a neighborhood of $\bar{G} \times \bar{G}$ such that

(i) $\Omega$ is of class $C^{\infty}$ off the diagonal of $G \times G$ :

(ii) there is a neighborhood of $\partial G \times G$ in $\bar{G} \times G$ in which $\bar{\partial}_{z} \Omega=$ 0 ;

(iii) if $g$ is a bounded $(0,1)$ form of class $C^{\infty}$ such that $\bar{\partial} g=0$ on $G$, and if

$$
f(z)=-\int_{G} g(\zeta) \wedge \Omega(\zeta, z)
$$

then $f \in C^{\infty}(G)$ and $\bar{\partial} f=g$ in $G$;

(iv) there is a constant $\Delta(G)$, independent of $z$ such that

$$
\int_{G}|a(\zeta, z)| d m(\zeta) \leqq \frac{\Delta(G)}{n}
$$

where $a(\zeta, z)$ is any coefficient of $\Omega$ and $d m$ is Lebesgue measure on $G$;

(v) $\bar{G}$ has a sequence $\left\{G_{\nu}\right\}$ of admissible neighborhoods whose intersection is $\bar{G}$ such that $\left\{\Delta\left(G_{\nu}\right)\right\}$ is a constant sequence.

Also, $G$ is the union of an increasing sequence of admissible open sets $\left\{G_{\nu}\right\}$ for which $\left\{\Delta\left(G_{\nu}\right)\right\}$ is a constant sequence. 
If $G$ is an open set in $C^{n}$ we denote by $B C^{\infty}(G)$ the space of functions on $G$ whose derivatives of all orders are bounded and continuous on $G$. We will need the following corollary of Theorem 2.1.

Corollary. Let $G$ be an admissible open set in $C^{n}$. Let $U_{k}$ be open in $\boldsymbol{C}^{n_{k}}, k=1,2$. Suppose that

$$
g_{1}(z, x, w), \cdots, g_{n}(z, x, w) \in B C^{\infty}\left(G \times U_{1} \times U_{2}\right)
$$

and that $g=\sum g_{j} d \bar{z}_{j}$ satisfies $\bar{\partial}_{z} g=0$ in $G \times U_{1} \times U_{2}$. Then there exists $f \in B C^{\infty}\left(G \times U_{1} \times U_{2}\right)$ such that

(i) $\bar{\partial}_{z} f=g$ in $G \times U_{1} \times U_{2}$;

(ii) $\|f\| \leqq \Delta(G) \max _{1 \leqq j \leqq n}\left\|g_{j}\right\|$;

(iii) if $D$ is a differential operator on $\boldsymbol{C}^{n_{1}} \times \boldsymbol{C}^{n_{2}}$ with constant coefficients, then

$$
\|D f\| \leqq \Delta(G) \max _{1 \leqq j \leqq n}\left\|D g_{j}\right\|
$$

(In particular, if each $g_{j}$ is holomorphic in $U_{2}$ for fixed $(z, x) \epsilon$ $G \times U_{1}$, then $f$ has the same property.)

Here $\|f\|=\sup _{G \times U_{1} \times U_{2}}|f|$.

Proof. Let $f(z, x, w)=-\int_{G} g(\zeta, x, w) \wedge \Omega(\zeta, z)$, where $\Omega$ is as in Theorem 2.1. Since all the derivatives of $g$ are bounded we may differentiate under the integral sign as often as we wish. The corollary then follows immediately from Theorem 2.1.

Let $G=G_{1} \times \cdots \times G_{r}$ be an open set in $C^{n}$ where each $G_{i}$ is an open set in $C^{n_{i}}$. If $f$ is a function on $G$ and $g=\sum g_{j} d \bar{z}_{j}$ is a $(0,1)$ form on $G$ we will use the following notation:

$$
\begin{aligned}
\|f\|_{G} & =\sup _{G}|f| \\
\|f\|_{G}^{(k, t)} & =\max _{\alpha \in A_{k, t}}\left\|\partial^{\alpha} f / \partial \bar{z}^{\alpha}\right\|_{G}
\end{aligned}
$$

(where $\alpha=\left(\alpha_{1}, \cdots, \alpha_{n}\right)$ is an $n$-tuple of nonnegative integers, $|\alpha|=$ $\sum \alpha_{j}$,

$$
\partial^{\alpha} f / \partial \bar{z}^{\alpha}=\partial^{|\alpha|} f / \partial \bar{z}_{1}^{\alpha_{1}} \cdots \partial \bar{z}_{n}^{\alpha},
$$

and

$$
\begin{aligned}
A_{k, t} & =\left\{\alpha=\left(\alpha_{1}, \cdots, \alpha_{n}\right):|\alpha| \leqq k \text { and } \alpha_{j}=0\right. \\
& \left.\left.\quad \text { if } j>n_{1}+\cdots+n_{t}\right\}\right) \\
\|f\|_{G}^{(k)} & =\|f\|_{G}^{(k, r)} \\
\|g\|_{G}^{(k, t)} & =\max _{1 \leqq j \leqq n}\left\|g_{j}\right\|^{(k, t)} \\
\|g\|_{G}^{(k)} & =\|g\|_{G}^{(k, r)} .
\end{aligned}
$$


We can now state our basic result.

THEOREM 2.2. Let $G=G_{1} \times \cdots \times G_{r}$ be an open set in $C^{n}$ where each $G_{i}$ is an admissible open set in $C^{n_{i}}$. Let $g$ be a $C^{\infty}(0,1)$ form in $G$ such that $\bar{\partial} g=0$ in $G$ and $\|g\|_{G}^{(r-1)}<\infty$. Then there exists $f \in$ $C^{\infty}(G)$ such that

(i) $\bar{\partial} f=g$ in $G$

(ii) $\|f\|_{G} \leqq(3 \Delta)^{r}\|g\|_{G}^{(r-1)}$.

Here $\Delta$ is any number $\geqq 1$ such that $\Delta \geqq \Delta\left(G_{i}\right)$ as defined in Theorem 2.1 .

Proof. We first prove the theorem in the case when each coefficient of $g$ is in $B C^{\infty}(G)$.

If $g$ is a $(0,1)$ form on $G$, then $g=\sum g^{i}$ where each $g^{i}$ is a $(0,1)$ form on $G_{i}$ with coefficients depending only on the other variables as parameters. For each $k, 1 \leqq k \leqq r$ we consider the following Assertion $k$ :

Let $G=G_{1} \times \cdots \times G_{r}$ be as above. Let $g$ be a $(0,1)$ form on $G$ whose coefficients lie in $B C^{\infty}(G)$ and such that $\bar{\partial} g=0$ in $G$. Suppose that

$$
g=\sum_{i=1}^{k} g^{i}
$$

where each $g^{i}$ is a $(0,1)$ form on $G_{i}$, (with coefficients depending also on the other variables). Then there exists $f \in B C^{\infty}(G)$ such that

(i) $\bar{\partial} f=g$

(ii) $\|f\|_{G} \leqq(3 \Delta)^{k}\|g\|^{(k-1, k-1)}$.

We shall prove Assertion 1 and then show that for $k=1,2, \cdots$, $r-1$, Assertion $k$ implies Assertion $k+1$. (Of course Assertion $r$ implies Theorem 2.2 in the case $g$ is of class $B C^{\infty}$.)

If $g$ satisfies the hypotheses of Assertion 1 , then $g$ is a $\bar{\partial}$-closed $(0,1)$ form in $G_{1}$ whose coefficients are holomorphic functions in $G_{2} \times$ $\cdots \times G_{r}$ for fixed $z$ in $G_{1}$. Assertion 1 thus follows directly from the Corollary to Theorem 2.1.

Suppose now that Assertion $k$ is true and that $g$ satisfies the hypotheses of Assertion $(k+1)$. Then

$$
g=\sum_{i=1}^{k+1} g^{i}
$$

Notice that $\bar{\partial} g=0$ implies $\bar{\partial}_{k+1} g^{k+1}=0$ (where $\bar{\partial}_{k+1}$ differentiates only with respect to the variables from $G_{k+1}$ ) and that the coefficients of $g$ are holomorphic in $G_{k+2} \times \cdots \times G_{r}$. Applying the corollary to Theorem 2.1 we conclude the existence of $u \in B C^{\infty}(G)$ such that $\bar{\partial}_{k+1} u=g^{k+1}$ and such that $u$ is holomorphic in $G_{k+2} \times \cdots \times G_{r}$. Let $s=g-\bar{\partial} u$. 
Then $\bar{\partial} s=\bar{\partial} g=0$ and $s$ is clearly a sum of $(0,1)$-forms involving only differentials in the variables from $G_{1}, \cdots, G_{k}$. By Assertion $k$ there exists $t \in B C^{\infty}(G)$ such that $\vec{\partial} t=s$.

Let $f=u+t$. Then $\bar{\partial} f=g$. Also,

$$
\begin{aligned}
\|f\| & \leqq\|u\|+\|t\| \\
& \leqq \Delta\|g\|+(3 \Delta)^{k}\|s\|^{(k-1, k-1)} .
\end{aligned}
$$

But

$$
\begin{aligned}
\|s\|^{(k-1, k-1)} \leqq & \left\|\sum_{i=1}^{k} g^{i}\right\|^{(k-1, k-1)} \\
& +\max _{1 \leqq j \leqq n_{k}}\left\|\partial u / \partial \bar{z}_{j}\right\|^{(k-1, k-1)} \\
\leqq & \|g\|^{(k-1, k-1)}+\|u\|^{(k, k)} \\
\leqq & \|g\|^{(k-1, k-1)}+\Delta\|g\|^{(k, k)} \\
\leqq & (2 \Delta)\|g\|^{(k, k)}
\end{aligned}
$$

Hence

$$
\begin{aligned}
\|f\| & \leqq\left(\Delta+(2 \Delta)(3 \Delta)^{k}\right)\|g\|^{(k, k)} \\
& \leqq(3 \Delta)^{k+1}\|g\|^{(k, k)}
\end{aligned}
$$

This concludes the proof in the case when $g$ is of class $B C^{\infty}$.

Suppose now that $g$ satisfies the hypotheses of Theorem 2.2. By Theorem 2.1 and what has been proved so far, we can find a sequence of open sets $\left\{G_{\nu}\right\}$ and a constant $C$ independent of $\nu$ such that

(i) $\bar{G}_{\nu} \subset G_{\nu+1} \subset G$

(ii) $G=\cup G_{\nu}$

(iii) there exists $f_{\nu} \in B C^{\infty}\left(G_{\nu}\right)$

such that $\bar{\partial} f_{\nu}=g$ on $G_{\nu}$ and

$$
\left\|f_{\nu}\right\|_{G_{\nu}} \leqq C\|g\|_{G}^{(r-1)} \text {. }
$$

For each $\mu$ let $S_{\mu}=\left\{f_{\nu} \mid G_{\mu}: \nu \geqq \mu\right\}$. Since $f_{\nu}-f_{\mu}$ is holomorphic on $G_{\mu}$ for each $\nu$, and $\left\{f_{\nu}-f_{\mu} \mid G_{\mu}\right\}$ is uniformly bounded, $S_{\mu}$ is relatively compact by Montel's theorem. Thus we may choose, for each $\mu$, a subsequence $\left\{f_{\nu, \mu}\right\}$ of $S_{\mu}$ which converges uniformly on $G_{\mu}$, such that $\left\{f_{\nu, \mu+1}\right\}$ is a subsequence of $\left\{f_{\nu, \mu}\right\}$. Then the diagonal sequence $\left\{f_{\mu, \mu}\right\}$ converges uniformly on each $G_{\mu}$ to a continuous function $f$ defined on all of $G$. But $f$ is in fact in $C^{\infty}(G)$ since, if $\nu>\mu, f_{\nu \nu}-f_{\mu \mu}$ is holomorphic on $G_{\mu}$ and $f_{\nu \nu}-f_{\mu \mu} \rightarrow f-f_{\mu \mu}$ on $G_{\mu}$. Thus $f-f_{\mu \mu}$ is holomorphic, hence in $C^{\infty}\left(G_{\mu}\right)$ so $f \in C^{\infty}\left(G_{\mu}\right)$ for each $\mu$. This also shows that $\bar{\partial} f=g$ on $G$. Finally, if $z \in G$ then there exists $\mu$ such that $z \in G_{\nu}$ for $\nu \geqq \mu$. This means that

$$
\left|f_{\mu \mu}(z)\right| \leqq C\|g\|_{G}^{(r-1)}
$$


but $f(z)=\lim f_{\mu \mu}(z)$ so

$$
\|f\|_{G} \leqq C\|g\|_{G}^{(r-1)}
$$

\section{Proofs of Theorems 1.1 and 1.2 .}

Proof of Theorem 1.1. We may suppose that $f$ has compact support in $U$. Choose $\phi \in C^{\infty}\left(C^{n}\right)$ such that $\phi \geqq 0, \int \phi=1$ and $\phi=0$ outside the closed unit ball. For each $\delta>0$ define $f_{\delta}$ by

$$
f_{\dot{o}}(z)=\int f(z-\delta w) \phi(w) d w
$$

Then $f_{\delta} \in C^{\infty}\left(C^{n}\right), f_{\grave{o}} \rightarrow f$ uniformly as $\delta \rightarrow 0$ and for each $\alpha$,

$$
\left(\partial^{\alpha} f_{\delta} / \partial \bar{z}^{\alpha}\right)(z)=\int\left(\partial^{\alpha} f_{\delta} / \partial \bar{z}^{\alpha}\right)(z-\delta w) \phi(w) d w
$$

so if $G$ is an open set in $C^{n}$,

$$
\left\|f_{\dot{o}}\right\|_{G}^{(s)} \leqq\|f\|_{G^{\delta}}^{(s)} \quad s=1,2, \cdots
$$

where $G^{\delta}=\{z-\delta w: z \in G,|w| \leqq 1\}$.

For each $i, 1 \leqq i \leqq r$ we can find a sequence $\left\{G_{i}^{*}\right\}$ of admissible neighborhoods such that $K_{i}=\cap G_{i}^{\nu}$ and such that $\left\{\Delta\left(G_{i}^{\nu}\right)\right\}$ is a constant sequence. Let us denote the constant by $\Delta_{i}$. Choose $\Delta \geqq 1$ such that $\Delta \geqq \Delta_{i}$ for $1 \leqq i \leqq r$.

Let $\varepsilon>0$ be given. Choose $\delta_{0}$ such that $\left\|f-f_{\tilde{\delta}}\right\|_{K}<\varepsilon / 2$ if $\delta<$ $\delta_{0}$. Choose $\nu$ such that if $G=G_{1}^{\nu} \times \cdots \times G_{r}^{\nu}$, then

$$
\|f\|_{G}^{(r)}<(3 \Delta)^{-r} \cdot \frac{\varepsilon}{4}
$$

Then there exists $\delta<\delta_{0}$ such that

$$
\|f\|_{\sigma^{\delta}}^{(r)}<(3 \Delta)^{-r} \frac{\varepsilon}{2}
$$

By Theorem 2.2 we can choose $u \in C^{\infty}(G)$ such that $\bar{\partial} u=\bar{\partial} f_{\delta}$ and

$$
\|u\|_{G} \leqq(3 \Delta)^{r}\left\|\bar{\partial} f_{\delta}\right\|_{G}^{(r-1)} \text {. }
$$

Then $h=f_{\delta}-u$ is holomorphic in a neighborhood of $K$ and

$$
\begin{aligned}
\|f-h\|_{K} & \leqq\left\|f-f_{\delta}\right\|_{K}+\left\|f_{\delta}-h\right\|_{K} \\
& <\varepsilon / 2+\|u\|_{K} \\
& <\varepsilon / 2+(3 \Delta)^{r}\left\|f_{\delta}\right\|_{G}^{r} \\
& <\varepsilon / 2+(3 \Delta)^{r}\|f\|_{G^{\delta}}^{r} \\
& <\varepsilon .
\end{aligned}
$$


Proof of Theorem 1.2. (Here we follow Lieb [8].) Since $K$ is compact we can choose finitely many neighborhoods $U_{x_{1}}, \cdots, U_{x_{m}}$ which cover $K$. Let $U=U_{x_{1}} \cup \cdots \cup U_{x_{m}}$. Choose sequences $\left\{G_{i}^{*}\right\}$ of admissible neighborhoods of $K_{i}, 1 \leqq i \leqq r$ as in the proof of Theorem 1.1 and let $\Delta$ be as above.

Let $\varepsilon>0$ be given. Choose $\nu$ such that $G^{\nu}=G_{1}^{\nu} \times \cdots \times G_{r}^{\nu}$ lies in $U$, and such that there exist holomorphic functions $h_{j}$ on $U_{x_{j}} \cap G^{\nu}$ with $\left\|f-h_{j}\right\|_{U_{x_{j}} \cap K}<\varepsilon$. Notice that $\left|h_{i}-h_{j}\right| \leqq\left|f-h_{i}\right|+\left|f-h_{j}\right|<$ $2 \varepsilon$ on $U_{x_{i}} \cap U_{x_{j}} \cap K$. By choosing $\nu$ larger if necessary we may suppose that $\left|h_{i}-h_{j}\right|<4 \varepsilon$ on $U_{x_{i}} \cap U_{x_{j}} \cap G^{\nu}$.

Choose a $C^{\infty}$ partition of unity $\phi_{1}, \cdots, \phi_{m}$ subordinate to the cover $\left\{U_{x_{i}}\right\}$. Let $g_{k}=\sum_{i} \phi_{i}\left(h_{k}-h_{i}\right)$. Notice that $\left\|g_{k}\right\|_{U x_{k} \cap K}<2 \varepsilon$. Also $g_{j}-g_{k}=$ $h_{j}-h_{k}$ and $\bar{\partial} g_{j}-\bar{\partial} g_{k}=0$ on $U_{x_{j}} \cap U_{x_{k}} \cap G^{\nu}$. Thus $\left\{\bar{\partial} g_{j}\right\}$ defines a $(0,1)$ form $g$ on $G^{\nu}$ which satisfies $\bar{\partial} g=0$ so by Theorem 2.2 we can find $u \in C^{\infty}\left(G^{\nu}\right)$ that $\bar{\partial} u=g$ on $G^{\nu}$ and

$$
\|u\|_{G^{\nu}} \leqq(3 \Delta)^{r}\|g\|_{G^{\nu}}^{(r-1)} .
$$

But

$$
\|g\|_{G^{\nu}}^{(r-1)} \leqq \max _{1 \leqq k \leqq n}\left\|g_{k}\right\|_{G^{\nu} \cap(r)}^{(\nu)} U_{x_{k}}
$$

and

$$
\partial^{\alpha} g_{k} / \partial \bar{z}^{\alpha}=\sum_{i}\left(\partial^{\alpha} \dot{\phi}_{i} / \partial \bar{z}^{\alpha}\right)\left(h_{k}-h_{i}\right) .
$$

Hence $\|g\|_{\sigma^{\nu}}^{(r-1)} \leqq 4 \varepsilon C$ where $C$ is a constant depending only on the partition of unity $\left\{\phi_{i}\right\}$.

Let $b_{j}=g_{j}-u$. Then $\bar{\partial} b_{j}=0$ on $U_{x_{j}} \cap G^{\nu}$ and $b_{j}-b_{k}=g_{j}-g_{k}=$ $h_{j}-h_{k}$. Thus $\left\{h_{j}-b_{j}\right\}$ defines a holomorphic function $h$ on $G^{\nu}$ and

$$
\begin{aligned}
\|f-h\|_{K} & \leqq \max _{1 \leqq j \leqq m}\left(\left\|f-h_{j}\right\|_{K}+\left\|g_{j}\right\|_{K}\right)+\|u\|_{K} \\
& \leqq \varepsilon+2 \varepsilon+4 C(3 \Delta)^{r} \varepsilon,
\end{aligned}
$$

where $C$ and $\Delta$ are independent of $\varepsilon$. Since $\varepsilon$ was arbitrary, this completes the proof.

4. Annihilating Measures for $H(K)$. If $G$ is an open set in $C^{n}$ we denote by $A^{(0,1)}(G)$ the space of differential forms of type $(0,1)$ on $G$ of class $C^{\infty}$. We topologize $A^{(0,1)}(G)$ as the direct sum of $n$ copies of the Frechet space $C^{\infty}(G)$ with the topology of uniform convergence on compact subsets of $G$ of derivatives of all orders. The dual space of $C^{\infty}(G)$ is the space of distributions on $C^{n}$ whose support is a compact subset of $G$. We will identify the dual space of $A^{(0,1)}(G)$ with the space of distributions with compact support in $G$. 
LeMmA 4.1. Let $G$ be a domain of holomorphy in $C^{n}, K$ a compact subset of $G$, and $\mu$ an annihilating measure for $H(K)$. Then there exist distributions $\lambda_{1}, \cdots, \lambda_{n}$ with compact support in $G$ such that $\mu=-\sum \partial \lambda_{j} / \partial \bar{z}_{j}$.

Proof. $C^{\infty}(G)$ and $A^{(0,1)}(G)$ are Frechet spaces. Since $G$ is a domain of holomorphy, $\bar{\partial}$ maps $C^{\infty}(G)$ onto the closed subspace of $(0,1)$ forms $g$ satisfying $\bar{\partial} g=0$. The measure $\mu$, considered as a continuous linear functional on $C^{\infty}(G)$, annihilates the kernel of $\bar{\partial}$, so by a theorem of Dieudonné and Schwartz [4], $\mu$ is in the image of the adjoint, $\bar{\partial}^{*}$, of $\bar{\partial}$. But if $\lambda=\left(\lambda_{1}, \cdots, \lambda_{n}\right)$ is an $n$-tuple of distributions with compact support in $G$ and $f \in C^{\infty}(G)$, then

$$
\begin{aligned}
\left(\bar{\partial}^{*} \lambda\right)(f) & =\lambda(\bar{\partial} f)=\sum \lambda_{j}\left(\partial f / \partial \bar{z}_{j}\right) \\
& =-\sum\left(\partial \lambda_{j} / \partial \bar{z}_{j}\right)(f) .
\end{aligned}
$$

Thus for some $\lambda_{1}, \cdots, \lambda_{n}$ we have $\mu=-\sum \partial \lambda_{j} / \partial \bar{z}_{j}$.

We will also consider, for a bounded open set $G$ in $C^{n}$, the Banach space $C^{r}(\bar{G})$ of continuous functions on $\bar{G}$ whose derivatives of order $\leqq r$ are bounded and continuous on $G$, with the norm

$$
\|f\|_{G}^{r}=\max _{|\alpha| \leqq r}\left\|D^{\alpha} f\right\|_{G}
$$

where $\alpha$ is a $2 n$-tuple of non-negative integers and

$$
D^{\alpha}=\left(\partial / \partial z_{1}\right)^{\alpha_{1}} \cdots\left(\partial / \partial z_{n}\right)^{\alpha_{n}}\left(\partial / \partial \bar{z}_{1}\right)^{\alpha_{n+1}} \cdots\left(\partial / \partial \bar{z}_{n}\right)^{\alpha_{2 n}} \cdot
$$

A continuous linear functional on $C^{r}(\bar{G})$ is easily seen to define a distribution on $C^{n}$ with support in $\bar{G}$. In addition, we will denote by $B_{r}^{(0,1)}(\bar{G})$ the space of $(0,1)$ forms on $G$ with coefficients in $C^{r}(\bar{G})$, topologized as the direct sum of $n$ copies of $C^{r}(\bar{G})$ with the norm

$$
\left\|\sum g_{j} d \bar{z}_{j}\right\|_{G}^{r}=\max _{1 \leqq j \leqq n}\left\|g_{j}\right\|_{G}^{r}
$$

If $G_{1} \subset G_{2}$ are two bounded open sets in $C^{n}$ let $R: B_{r}^{(0,1)}\left(G_{2}\right) \rightarrow$ $B_{r}^{(0,1)}\left(G_{1}\right)$ be the operator which restricts forms in $G_{2}$ to $G_{1}$. Then $R^{*}$ is a norm-decreasing embedding of the dual space of $B_{r}^{(0,1)}\left(G_{1}\right)$ into $B_{r}^{(0,1)}\left(G_{2}\right)^{*}$ since, if $\lambda \in B_{r}^{(0,1)}\left(G_{1}\right)^{*}$, and $g=\sum g_{j} d \bar{z}_{j}$ is in $B_{r}^{(0,1)}\left(G_{2}\right)$,

$$
\begin{aligned}
\left|\left(R^{*} \lambda\right)(g)\right| & =|\lambda(R g)| \leqq\|\lambda\|\|R g\|_{G_{1}}^{r} \\
& \leqq\|\lambda\|\|g\|_{G_{2}}^{r},
\end{aligned}
$$

so $\left\|R^{*} \lambda\right\| \leqq\|\lambda\|$.

With these preliminaries we can proceed to the proof of Theorem 1.3.

Proof of Theorem 1.3. Let $\left\{G_{\nu}\right\}$ be a sequence of bounded open 
neighborhoods of $K$ such that

(i) $\bar{G}_{\nu} \subset G_{\nu-1}$

(ii) $K=\cap G_{\nu}$

(iii) there exists a constant $C$ independent of $\nu$ such that if $g \in$ $A^{(0,1)}\left(G_{\nu}\right), \bar{\partial} g=0$ in $G_{\nu}$, and $\|g\|_{G^{\nu}}^{(r-1)}<\infty$, then there exists $f \in C^{\infty}\left(G_{\nu}\right)$ such that $\bar{\partial} f=g$ and

$$
\|f\|_{G_{\nu}} \leqq C\|g\|_{G^{\nu}}^{(r-1)}
$$

If $\mu$ is an annihilating measure for $H(K)$ we can apply Lemma 4.1 to obtain, for each $\nu$, an $n$-tuple $\lambda^{\nu}=\left(\lambda_{1}^{\nu}, \cdots, \lambda_{n}^{\nu}\right)$ of distributions with compact support in $G_{\nu}$ such that $\mu=-\sum \partial \lambda_{j}^{\nu} / \partial \bar{z}_{j}$. Let $W_{\nu}$ be the subspace of $C^{r-1}\left(\bar{G}_{\nu}\right)$ consisting of restrictions to $G_{\nu}$ of $C^{\infty}$ functions on $C^{n}$. If $f \in W_{\nu}$ we can find $h \in C^{\infty}\left(G_{\nu}\right)$ such that $f-h$ is holomorphic on $G_{\nu}$ and $\|h\|_{G_{\nu}} \leqq C\|\bar{\partial} f\|_{G_{\nu}}^{(r-1)}$ where $C$ is the constant in (iii) above. Thus

$$
\begin{aligned}
\left|\lambda^{\nu}(\bar{\partial} f)\right| & =\left|\int f d \mu\right|=\left|\int h d \mu\right| \\
& \leqq\|\mu\|\|h\|_{K} \leqq C\|\mu\|\|\bar{\partial} f\|_{G^{\nu}}^{\left(\gamma^{2}-1\right)}
\end{aligned}
$$

where $\|\mu\|$ is the total variation of $\mu$. This means that $\lambda^{\nu}$ defines a continuous linear functional on the subspace $\bar{\partial} W_{\nu}$ of $B_{r-1}^{(0,1)}\left(G_{\nu}\right)$ of norm $\leqq C\|\mu\|$. By the Hahn-Banach theorem there is an $n$-tuple, which we will continue to denote by $\lambda^{2}$, of continuous linear functionals on $C^{r-1}\left(\bar{G}_{\nu}\right)$ such that

(a) $\lambda^{\nu}(\bar{\partial} f)=\int f d \mu$ for all $f \in C^{\infty}\left(C^{n}\right)$

(b) $\left\|\lambda^{\nu}\right\| \leqq C\|\mu\|$.

Now, by composing with the adjoint of the appropriate restriction operator we may consider each $\lambda^{\nu}$ so obtained as a continuous linear functional on $B_{r-1}^{(0,1)}\left(G_{1}\right)$. Then the sequence $\left\{\lambda^{2}\right\}$ constitutes a bounded sequence of elements in the dual space of a separable Banach space. Consequently, there is a subsequence $\left\{\lambda^{\nu \prime}\right\}$ and an $n$-tuple $\lambda=$ $\left(\lambda_{1}, \cdots, \lambda_{n}\right)$ of continuous linear functionals on $C^{r-1}\left(\bar{G}_{1}\right)$ such that $\lambda^{\nu^{\prime}} \rightarrow \lambda$ in the weak star topology. Since each $\lambda_{j}^{\nu^{\prime}}$ is supported, as a distribution, on $\bar{G}_{\nu}$, and since $K=\cap G_{\nu}$, it follows that the support of each $\lambda_{j}$, as a distribution, lies in $K$. Moreover, if $f$ is a $C^{\infty}$ function on $\boldsymbol{C}^{n}$ then

$$
\lambda(\bar{\partial} f)=\lim \lambda^{\nu^{\prime}}(\bar{\partial} f)=\int f d \mu,
$$

i.e., $\mu=-\sum \partial \lambda_{j} / \partial \bar{z}_{j}$. Finally, it is clear that each $\lambda_{j}$ is of order $\leqq r-1$.

Conversely, suppose $\mu$ is a measure on $K$, and $\mu=\sum \partial \lambda_{j} / \partial \bar{z}$, where $\lambda_{1}, \cdots, \lambda_{n}$ are distributions with compact support on $K$. If $f$ is holo- 
morphic in a neighborhood of $K$, then $\partial f / \partial \bar{z}_{j}, 1 \leqq j \leqq n$, are identically 0 in a neighborhood of $K$ so

$$
\begin{aligned}
\int f d \mu & =\sum\left(\partial \lambda_{j} /\left(\partial \bar{z}_{j}\right)(f)\right. \\
& =-\sum \lambda_{j}\left(\partial f / \partial \bar{z}_{j}\right) \\
& =0
\end{aligned}
$$

since the $\lambda_{j}$ are supported on $K$.

Proof of Theorem 1.4. Let $\left\{\phi_{i}\right\}$ be a $C^{\infty}$ partition of unity subordinate to the open covering $\left\{U_{i}\right\}$, i.e., suppose $\phi_{i} \in C^{\infty}\left(U_{i}\right), \phi_{i}$ has compact support, $0 \leqq \phi_{i} \leqq 1$, and $\sum \phi_{i}=1$ on a neighborhood of $K$. If $\mu$ is an annihilating measure for $H(K)$, then by Theorem 1.3 there exist measures $\lambda_{1}, \cdots, \lambda_{n}$ on $K$ such that

$$
\begin{aligned}
\mu & =-\sum \partial \lambda_{j} / \partial \bar{z}_{j}=-\sum_{j} \partial\left(\sum_{i} \phi_{i} \lambda_{j}\right) / \partial \bar{z}_{j} \\
& =-\sum_{j} \sum_{i} \phi_{i}\left(\partial \lambda_{j} / \partial \bar{z}_{j}\right)-\sum_{j} \sum_{i}\left(\partial \phi_{i} / \partial \bar{z}_{j}\right) \lambda_{j} \\
& =\sum_{i}\left\{\phi_{i} \mu-\sum_{j}\left(\partial \phi_{i} / \partial \bar{z}_{j}\right) \lambda_{j}\right\} \\
& \equiv \sum_{i} \mu_{i}
\end{aligned}
$$

where each $\mu_{i}$ is a measure compactly supported on $U_{i} \cap K$. If $h$ is holomorphic on $U_{i} \cap K$, then

$$
\partial\left(h \phi_{i}\right) / \partial \bar{z}_{j}=h\left(\partial \phi_{i} / \partial \bar{z}_{j}\right)
$$

for $1 \leqq j \leqq n$. Thus

$$
\begin{aligned}
\int h d \mu_{i} & =\int h \phi_{i} d \mu-\sum_{j} \int h\left(\partial \dot{\phi}_{i} / \partial \bar{z}_{j}\right) d \lambda_{j} \\
& =\int h \phi_{i} d \mu-\sum_{j} \int\left(\partial / \partial \bar{z}_{j}\right)\left(\phi_{i} h\right) d \lambda_{j} \\
& =0 .
\end{aligned}
$$

\section{REFERENCES}

1. A. Andreotti and W. Stoll, The extension of bounded holomorphic functions from hypersurfaces in a polycylinder, Rice University Studies, 56 No. 2 (1970), 199-222.

2. A. Browder, Introduction to Function Algebras, W. A. Benjamin, Inc., New York, 1969.

3. E. M. Chirka, Approximation by holomorphic functions on smooth manifolds in $\boldsymbol{C}^{n}$, Math. USSR Sbornik 7 (1969), 95-114 (translated from Mat. Sbornik, 78 (120) (1969) No. 1). 4. J. Dieudonné and L. Schwartz, La dualité dans les espaces $(F)$ et $(L F)$, Annales Inst. Fourier, 1 (1949), 61-101. 
5. H. Grauert and I. Lieb, Das Ramirezsche Integral und die Lösung der Gleichung $\overline{\partial f}=a$ im Bereich der beschränkten Formen, Rice University Studies, 56 No. 2 (1970), $29-50$.

6. E. Kallin, A non-local function algebra, Proc. Nat. Acad. Sciences, 49 (1963), 821824.

7. N. Kerzman, Hölder and $L^{p}$ estimaties for solutions of $\bar{\partial} u=f$ in strongly pseudoconvex domains, Comm. Pure Appl. Math., 24 (1971), 301-379.

8. I. Lieb, Ein Approximationssatz auf streng pseudoconvexen Gebieten, Math. Ann., 184 (1969), 56-60.

Received September 22, 1971 and in revised form February 3, 1972. This research was supported by NSF Grants GP-11969 and GP-21326 at Brown University.

BROWN UNIVERSITY 


\section{PACIFIC JOURNAL OF MATHEMATICS}

\section{EDITORS}

\author{
H. SAMELSON \\ Stanford University \\ Stanford, California 94305 \\ C. R. HOBBY \\ University of Washington \\ Seattle, Washington 98105
}

\section{J. DugundjI}

Department of Mathematics University of Southern California Los Angeles, California 90007

\section{RICHARD ARENS}

University of California

Los Angeles, California 90024

\section{ASSOCIATE EDITORS}
E. F. BECKENBACH
B. H. NeumanN
F. WOLF
K. YosHIDA

\section{SUPPORTING INSTITUTIONS}

\author{
UNIVERSITY OF BRITISH COLUMBIA \\ CALIFORNIA INSTITUTE OF TECHNOLOGY \\ UNIVERSITY OF CALIFORNIA \\ MONTANA STATE UNIVERSITY \\ UNIVERSITY OF NEVADA \\ NEW MEXICO STATE UNIVERSITY \\ OREGON STATE UNIVERSITY \\ UNIVERSITY OF OREGON \\ OSAKA UNIVERSITY
}

\author{
UNIVERSITY OF SOUTHERN CALIFORNIA \\ STANFORD UNIVERSITY \\ UNIVERSITY OF TOKYO \\ UNIVERSITY OF UTAH \\ WASHINGTON STATE UNIVERSITY \\ UNIVERSITY OF WASHINGTON \\ ${ }^{*} \stackrel{*}{*}{ }^{*}$
AMERICAN MATHEMATICAL SOCIETY
NAVAL WEAPONS CENTER
}

The Supporting Institutions listed above contribute to the cost of publication of this Journal, but they are not owners or publishers and have no responsibility for its content or policies.

Mathematical papers intended for publication in the Pacific Journal of Mathematics should be in typed form or offset-reproduced, (not dittoed), double spaced with large margins. Underline Greek letters in red, German in green, and script in blue. The first paragraph or two must be capable of being used separately as a synopsis of the entire paper. The editorial "we" must not be used in the synopsis, and items of the bibliography should not be cited there unless absolutely necessary, in which case they must be identified by author and Journal, rather than by item number. Manuscripts, in duplicate if possible, may be sent to any one of the four editors. Please classify according to the scheme of Math. Rev. Index to Vol. 39. All other communications to the editors should be addressed to the managing editor, Richard Arens, University of California, Los Angeles, California, 90024.

50 reprints are provided free for each article; additional copies may be obtained at cost in multiples of 50 .

The Pacific Journal of Mathematics is published monthly. Effective with Volume 16 the price per volume (3 numbers) is $\$ 8.00$; single issues, $\$ 3.00$. Special price for current issues to individual faculty members of supporting institutions and to individual members of the American Mathematical Society: $\$ 4.00$ per volume; single issues $\$ 1.50$. Back numbers are available.

Subscriptions, orders for back numbers, and changes of address should be sent to Pacific Journal of Mathematics, 103 Highland Boulevard, Berke'ey, California, 94708.

PUBLISHED BY PACIFIC JOURNAL OF MATHEMATICS, A NON-PROFIT CORPORATION

Printed at Kokusai Bunken Insatsusha (International Academic Printing Co., Ltd.), 270, 3-chome Totsuka-cho, Shinjuku-ku, Tokyo 160, Japan. 


\section{Pacific Journal of Mathematics}

\section{Vol. 43, No. $3 \quad$ May, 1972}

Max K. Agoston, An obstruction to finding a fixed point free map on a manifold.... 543

Nadim A. Assad and William A. Kirk, Fixed point theorems for set-valued mappings

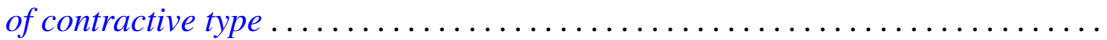

John Winston Bunce, Characterizations of amenable and strongly amenable

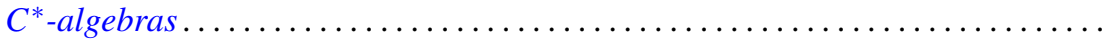

Erik Maurice Ellentuck and Alfred Berry Manaster, The decidability of a class of

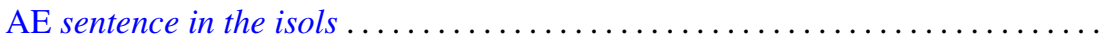

U. Haussmann, The inversion theorem and Plancherel's theorem in a Banach

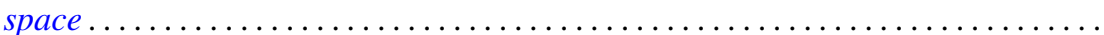

Peter Lawrence Falb and U. Haussmann, Bochner's theorem in infinite dimensions.

Peter Fletcher and William Lindgren, Quasi-uniformities with a transitive base ..... Dennis Garbanati and Robert Charles Thompson, Classes of unimodular abelian

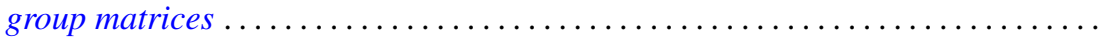

Kenneth Hardy and R. Grant Woods, On c-realcompact spaces and locally bounded normal functions

Manfred Knebusch, Alex I. Rosenberg and Roger P. Ware, Grothendieck and Witt rings of hermitian forms over Dedekind rings .......................

George M. Lewis, Cut loci of points at infinity.

Jerome Irving Malitz and William Nelson Reinhardt, A complete countable $L_{\omega_{1}}^{Q}$ theory with maximal models of many cardinalities . . . . . . . . . . . . . . . . .

Wilfred Dennis Pepe and William P. Ziemer, Slices, multiplicity, and Lebesgue

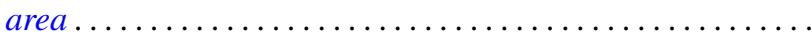

Keith Pierce, Amalgamating abelian ordered groups . .

Stephen James Pride, Residual properties of free groups . . . . . . . . . . . . . 725

Roy Martin Rakestraw, The convex cone of n-monotone functions .

T. Schwartzbauer, Entropy and approximation of measure preserving transformations .

Peter F. Stebe, Invariant functions of an iterative process for maximization of a polynomial...

Kondagunta Sundaresan and Wojbor Woyczynski, L-orthogonally scattered

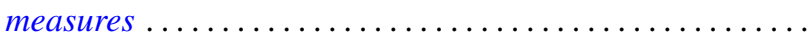

Kyle David Wallace, $C_{\lambda}$-groups and $\lambda$-basic subgroups $\ldots \ldots \ldots$

Barnet Mordecai Weinstock, Approximation by holomorphic functions on certain

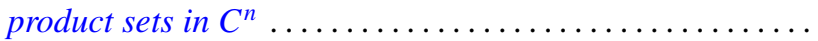

Donald Steven Passman, Corrections to: "Isomorphic groups and group rings”.

Don David Porter, Correction to: "Symplectic bordism, Stiefel-Whitney numbers, and a Novikov resolution"

John Ben Butler, Jr., Correction to: “Almost smooth perturbations of self-adjoint operators".

Constantine G. Lascarides, Correction to: "A study of certain sequence spaces of Maddox and a generalization of a theorem of Iyer" ...... ...

George A. Elliott, Correction to: "An extension of some results of takesaki in the reduction theory of von neumann algebras" ......................... 